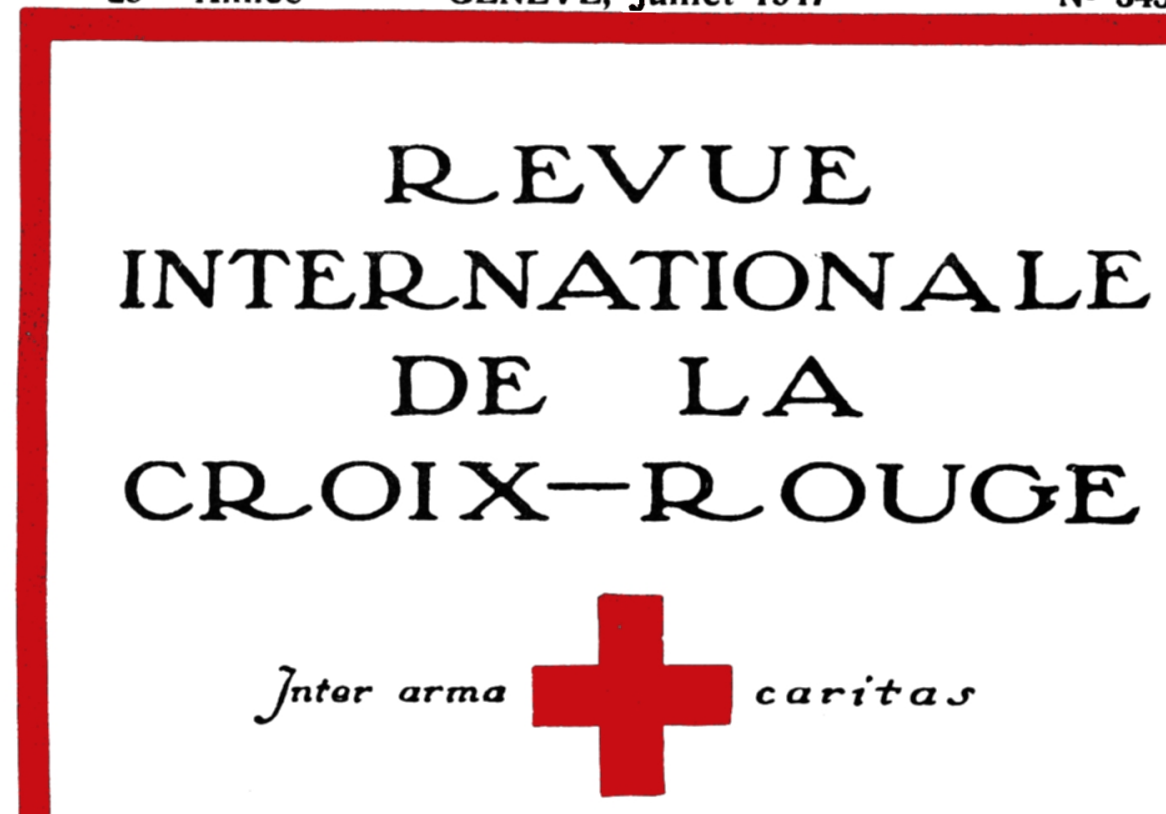

\title{
BULLETIN INTERNATIONAL DES SOCIÉTÉS DE LA CROIX-ROUGE
}

\author{
paraissant à la fin de chaque mois
}

T. LXXVIII, N' 539

\section{SOMMAIRE :}

COMITE INTERNATIONAL DE LA CROIX-ROUGE. - Réunion de Commissions de la Croix-Rouge internationale à Paris (mai 1947): Commission spéciale d'etude chargée d'examiner les moyens d'accroître l'efficacité de l'action du Comité international de la Croix-Rouge, 541; Commission permanente de la Conférence internationale de la Croix-Rouge, 542. - Entretiens des 9-12 juin avec des experts gouvernementaux, pour l'étude des Conventions protégeant les victimes de la guerre, 543. - Rapport sommaire sur les travaux de la Conférence d'experts gouvernementaux pour l'étude des Conventions protégeant les vietimes de la guerre, 544. - Activité du Comité international dans la région du Pacifique, 598. - Visites de camps de prisonniers de guerre, détachements, trains de rapatriement, hôpitaux et prisons, faites par des délégués du Comité international (hors-texte), 599. Chronique de l'Agence centrale des prisonniers de guerre, 599.

Abbé Henri MARMIER, professeur au Grand Séminaire de Fribourg. Le Saint-Siège et son œuvre d'assistance en faveur des victimes de la guerre (1939-1945) (horstexte), 602.

BIBLIOGRAPHIE. - A travers les revues, 614.

BULLETIN INTERNATIONAL DES SOCIÉTÉS DE LA CROIX-ROUGE.

(Voir la $4^{\mathrm{e}}$ page de la couverture).

\section{GENĖVE}

\section{COMITÉ INTERNATIONAL DE LA CROIX-ROUGE}




\section{Comité international de la Croix-Rouge à Genève}

MM. Burckhardt, Carl J., docteur en philosophie, professeur honorairc de l'Université de Zurich, professeur ordinaire à l'Institut universitaire de Hautes Etudes internationales à Genève, ancien Haut-Commissaire de la Société des Nations. - (I933) ${ }^{1}$, président (en congé).

Gloor, Ernest, docteur en médecine (1945), vice-président.

Bodmer, Martin, (I940), vice-président.

Chenevière, Jacques, docteur ès lettres h. c. (IgI9).

Huber, Max, docteur en droit, ancien président de la Cour permanente de justice internationale (I923), président d'honneur.

Mile Ferrière, Suzanne, directrice-adjointe de l'International Social Service (1924).

M. Patry, Georges, docteur en médecine, colonel, ancien médecin de division de l'armée suisse (I929).

M11es Odier, Lucie, ex-chef du Service des infirmières-visiteuses de la Croix-Rouge genevoise (1930).

Bordier, Renée, ancienne infirmière-chef au "Bon Secours" (I938).

MM. Cramer, Alec, docteur en médecine, colonel (1938).

Chapuisat, Edouard, docteur ès lettres h. c. (I938).

Etter, Philippe, conseiller fédéral (I940).

Vischer, Adolf-L., docteur en médecine (I945).

van Berchem, René, docteur en droit, banquier (I946).

Schindler, Dietrich, docteur en droit, professeur à l'Université de Zurich (1946).

Boissier, Léopold, docteur en droit, professeur à l'Université de Genève, secrétaire général de l'Union interparlementaire (I946).

Grasset, Edmond, docteur en médecine, professeur à l'Université de Genève, directeur de l'Institut d'hygiène (I946).

Lüchinger, Adolf, docteur en droit, président de la ville de Zurich (I946).

Carry, Paul, docteur en droit, professeur à l'Université de Genève (1946).

I Les années indiquées dans les parenthèses désignent les dates de nomination des membres du Comité international. 

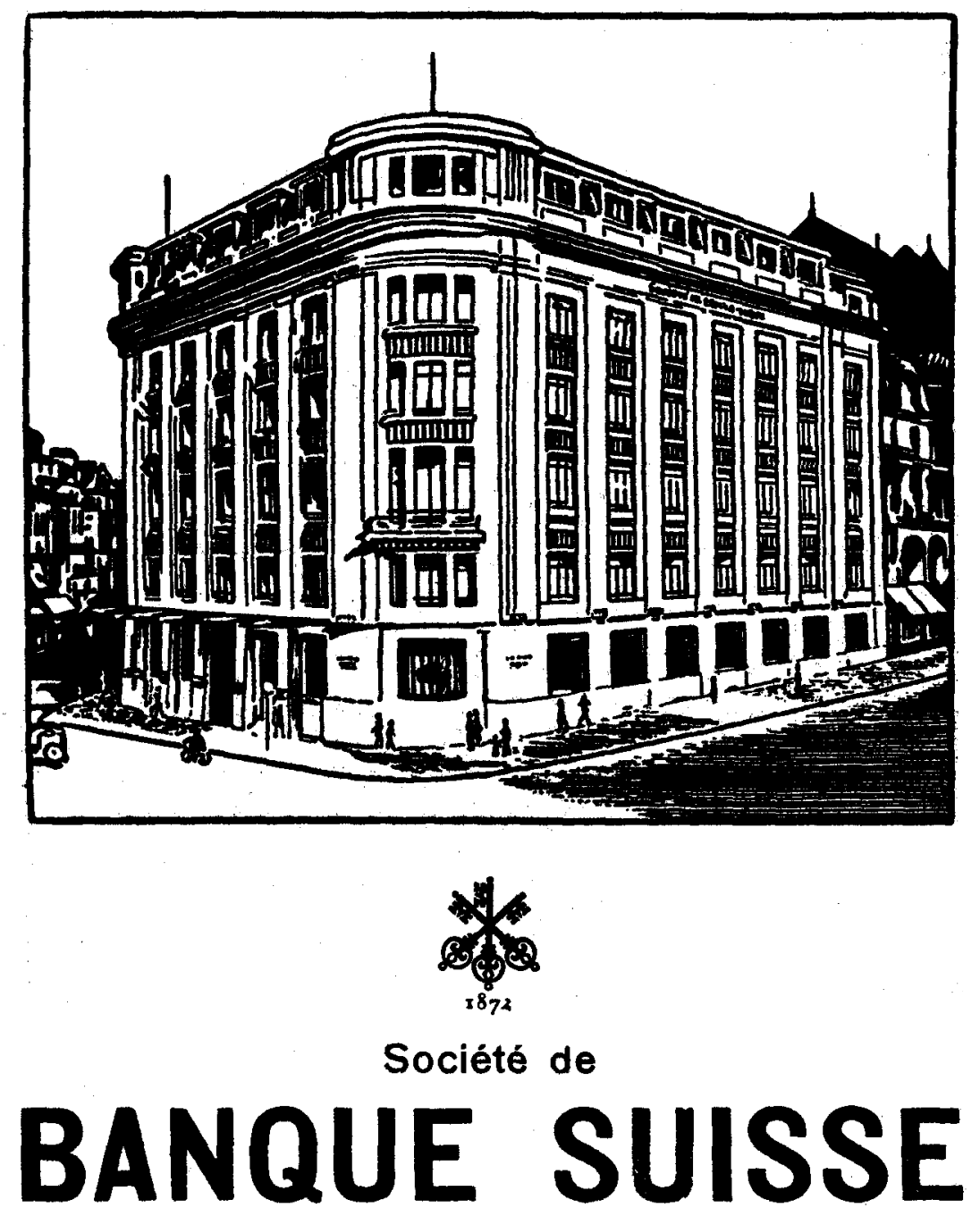

BALE *ZURICH * ST-GALL* LAUSANNE NEUCHATEL * CHAUX-DE-FONDS * SCHAFFHOUSE LONDRES * NEW-YORK

\section{SIËGE DE GENÈVE}

2 , rue de la Confédération

Capital-actions et réserves: Fr. 195 millions 


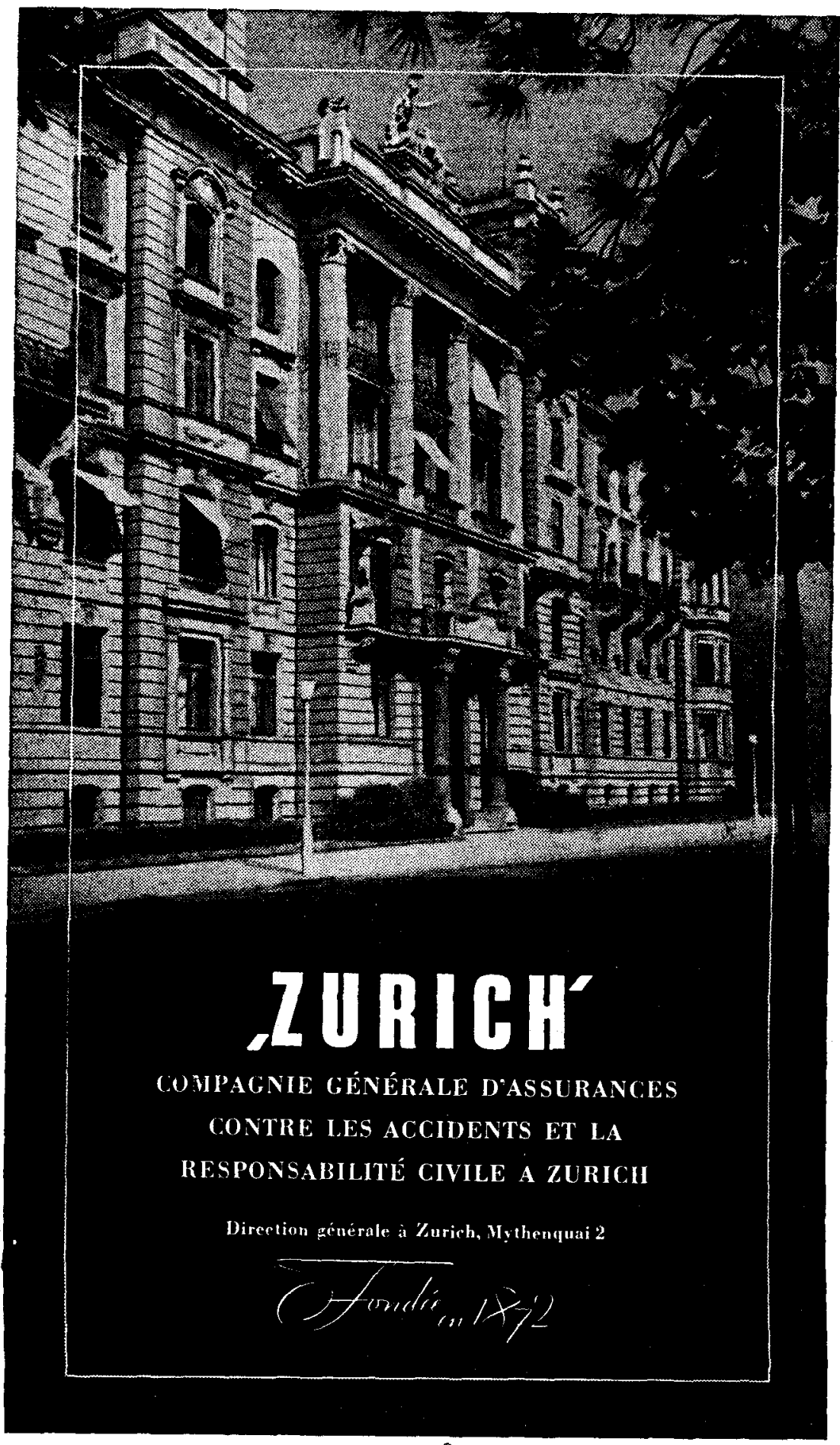




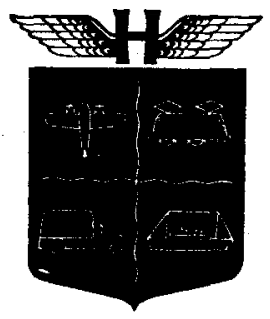

Compagnie d'Assurances Générales

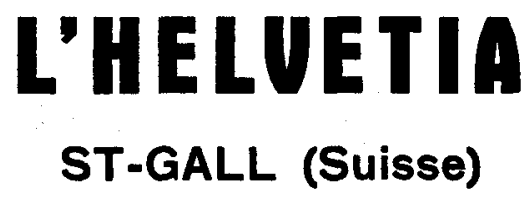

Fondere en 1858

ASSURANGES TRAMSPORTS

et

REASSURAlices dans toutos les Branchos

$\begin{array}{ll} & \text { Francs sulsses } \\ \text { Capital social (2/s versé) } & 10.000 .000 \\ \text { Total des Réserves } & 24.185 .000 \\ \text { Primes brutes } 1946 & 19.116 .115\end{array}$




\section{Société Fiduciaire Romande OFOR S.A.}

Blsqe a Aenére, Lue a'Italle 9 - Tel. 48291 Fondée en 1933

Membre de la Chambre Sulsse pour Expertises Comptables

\section{Toutes fonctions fiduoiaires}

Expertises - Revisions facultatives ou imposees par la lol Controle bancaire - Visa d'affidavits - Organisations comptables Impôts - Déclarations et recours par spécialiste Administration, domiciliation et liquidation de sociétés.
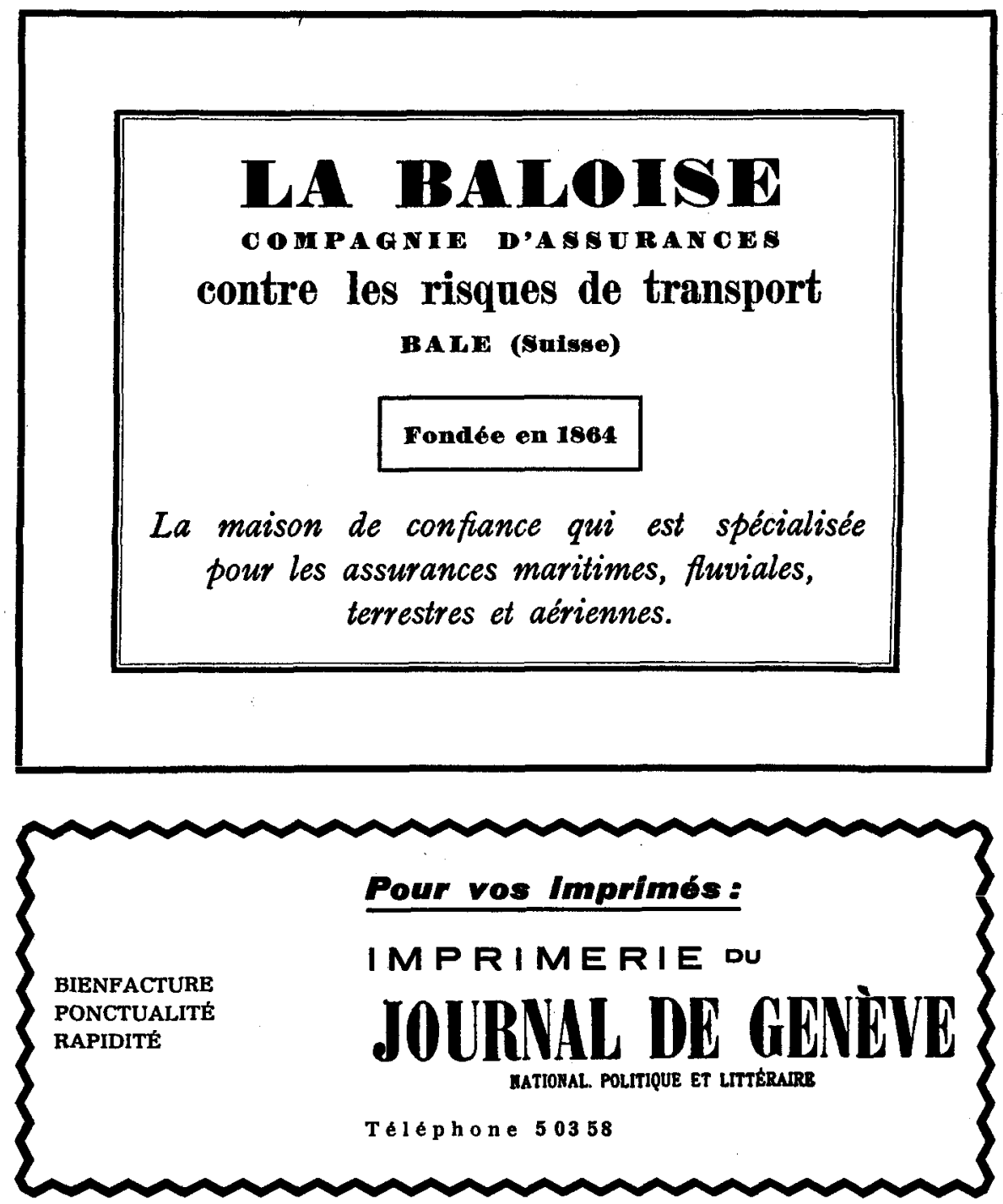


\section{Buffets}

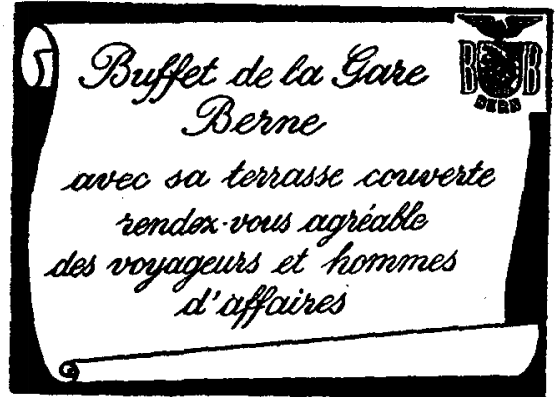

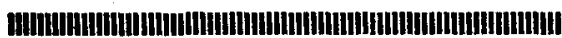

De passage à

\section{ZURICH}

rendez-vous au BUFFET DE LA GARE vous y trouverez l'accueil le plus aimable une bonne table un service prompt et soigné

Vous screz reçus et soignés impeccablement

Mêmes maisons :

PARK HOTEL, Vitznau (avril-nov.) SUVRETTA HOUSE, St-Moritz (Engadine) (Saisons été et hiver)

\section{Hôtels}

H O T E L

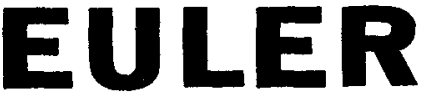

Ier R A N G

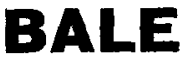

En face de la Gare:CFF

$$
\begin{array}{rr}
\text { Téléphone } & \text { Télégramme } \\
21723 & \text { Eulerbơtel }
\end{array}
$$

\section{LA RÉSIDENCE}

Florissant, 11

T6́l. 41388 (8 lignes) GENÈVE

\section{HOTEL - RESTAURANT - BAR}

Grands et petits salons pour réceptions 160 Iits 50 salles de bains

Téléphone dans toutes les chambres

Doux tennis - Parc pour autos

Arrangements pour familles 


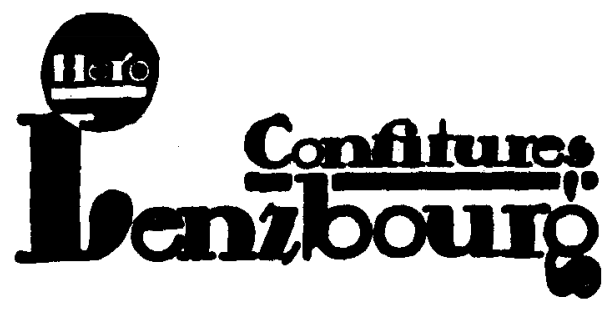

\section{SALVAJ \& CE S.A.}

AGENCE DE GROS

DENRÉES ALIMENTAIRES ET MATIERES PREMIËRES

Importation

Exportation

*

Rue Ad.-Lachenal, 3 - GENĖVE

LES

PORTE-MINES

\begin{tabular}{|c|c|c|c|c|}
\hline & $C$ & & $D$ & \\
\hline$C$ & $A$ & $R$ & $A$ & $N$ \\
\hline & $R$ & & $C$ & \\
\hline$D$ & $A$ & $C$ & $H$ & $E$ \\
\hline & $N$ & & $E$ & \\
\hline
\end{tabular}

SON T

MEILLEURS

Les matlères éprouvées pour la confection des prothéses, couronnes et bridges

\section{PaLAdoOi et PaLAPONIT \\ onse revent}

EN VENTE DANS tOUS LES DEPOTS DENTAIRES LEUKON S. A.s Obere Zäune, 10 - ZURICH 


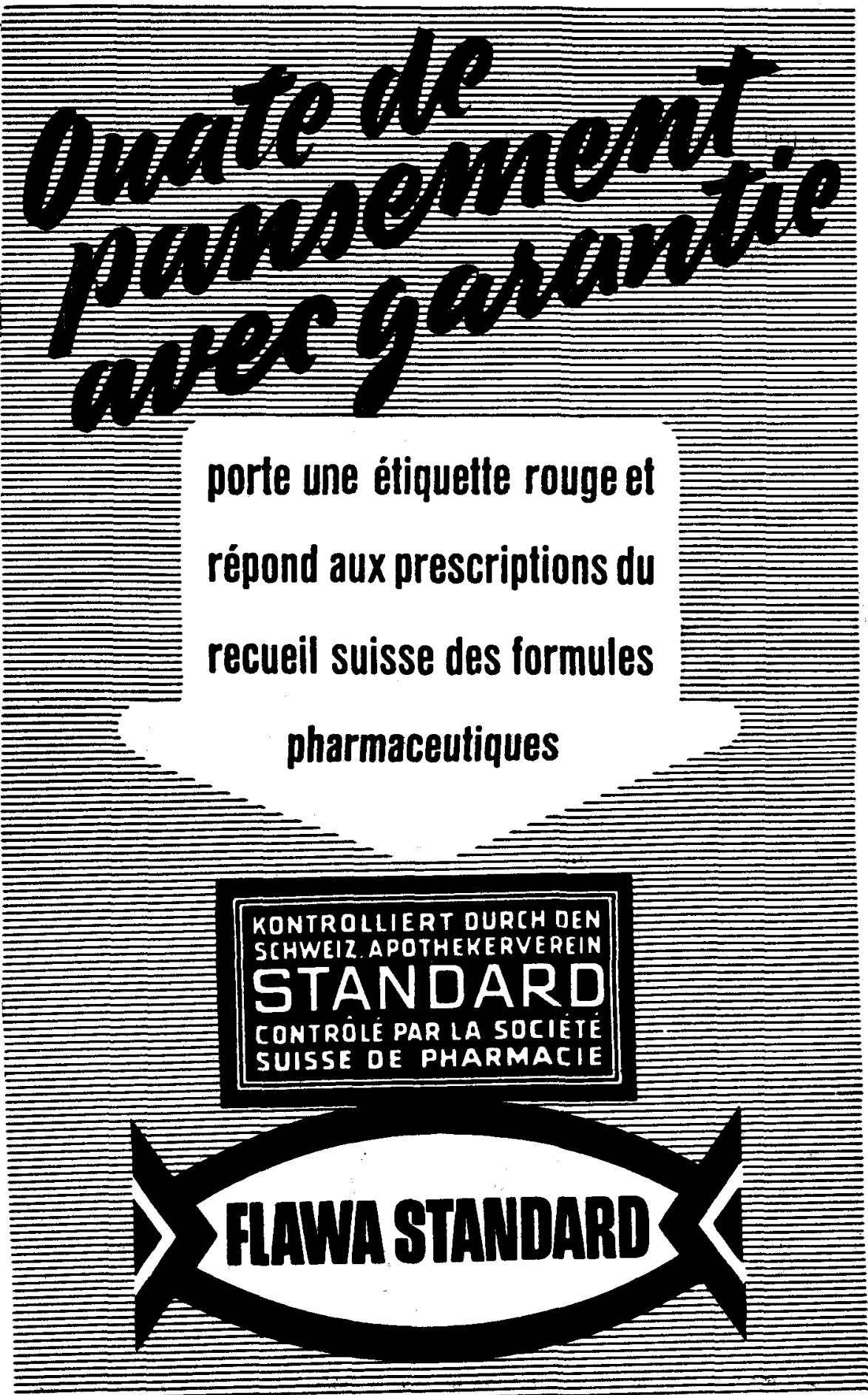

FLA W A Fabriques suisses d'objets de pansement et d'ouates S.A., Flawil 


\section{AUDERSET - DUBOIS}

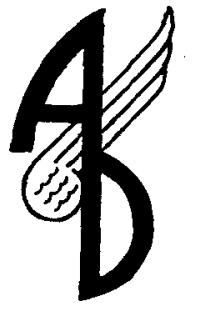

10, RUE DEB aUiB. TELEPHONE 20420

TRANSPORTS DÉMÉNAGEMENTS EXOURSIONS

GENEVE
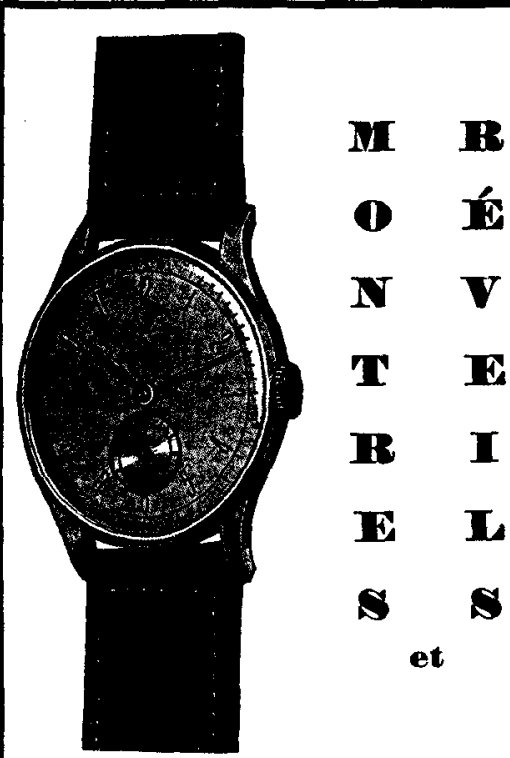

Fabrique d'Horlogerie N U R $\mathbf{~} \mathbf{D}$

Erwin Triebold, Mumpr ( $S U$ ISSE)
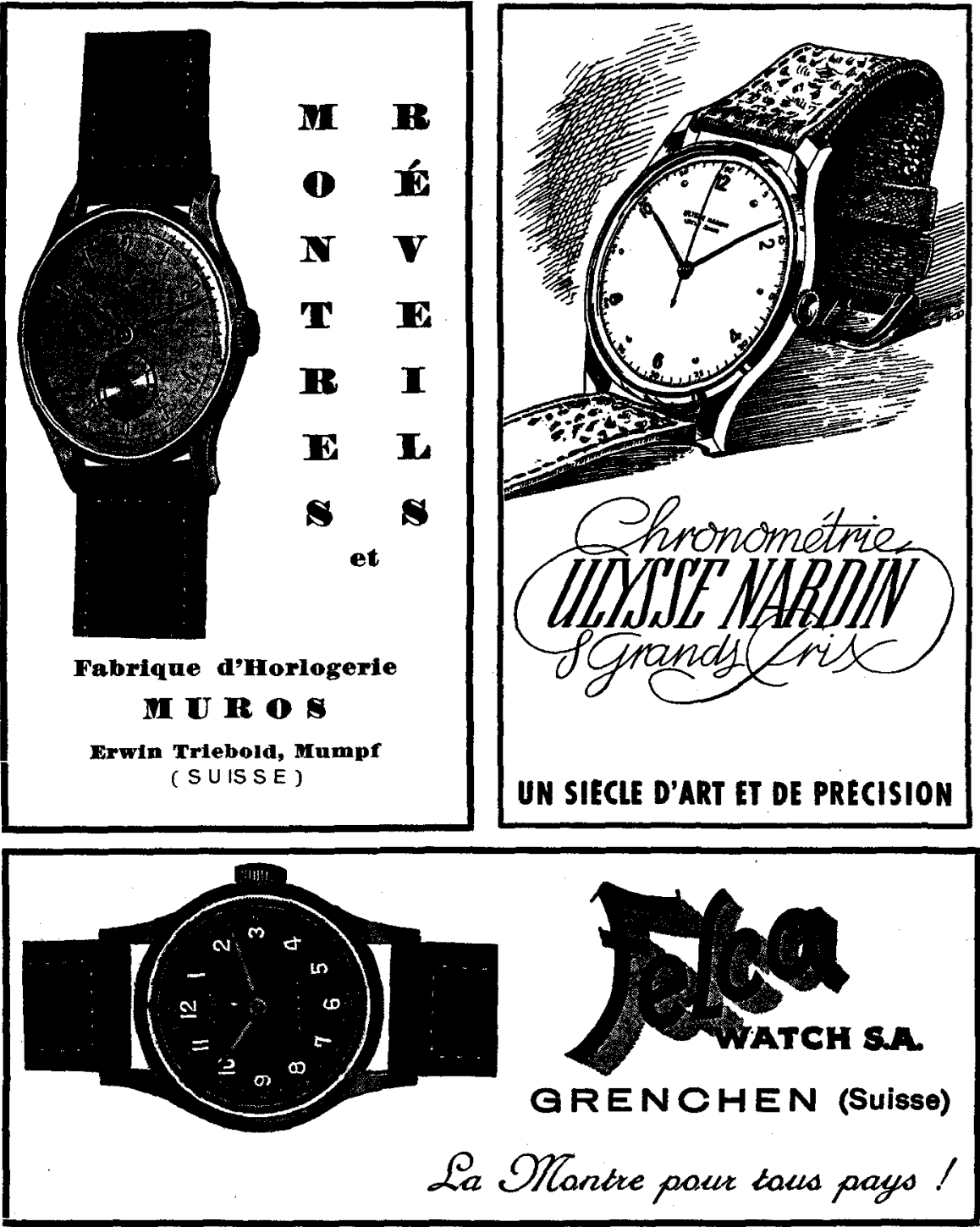


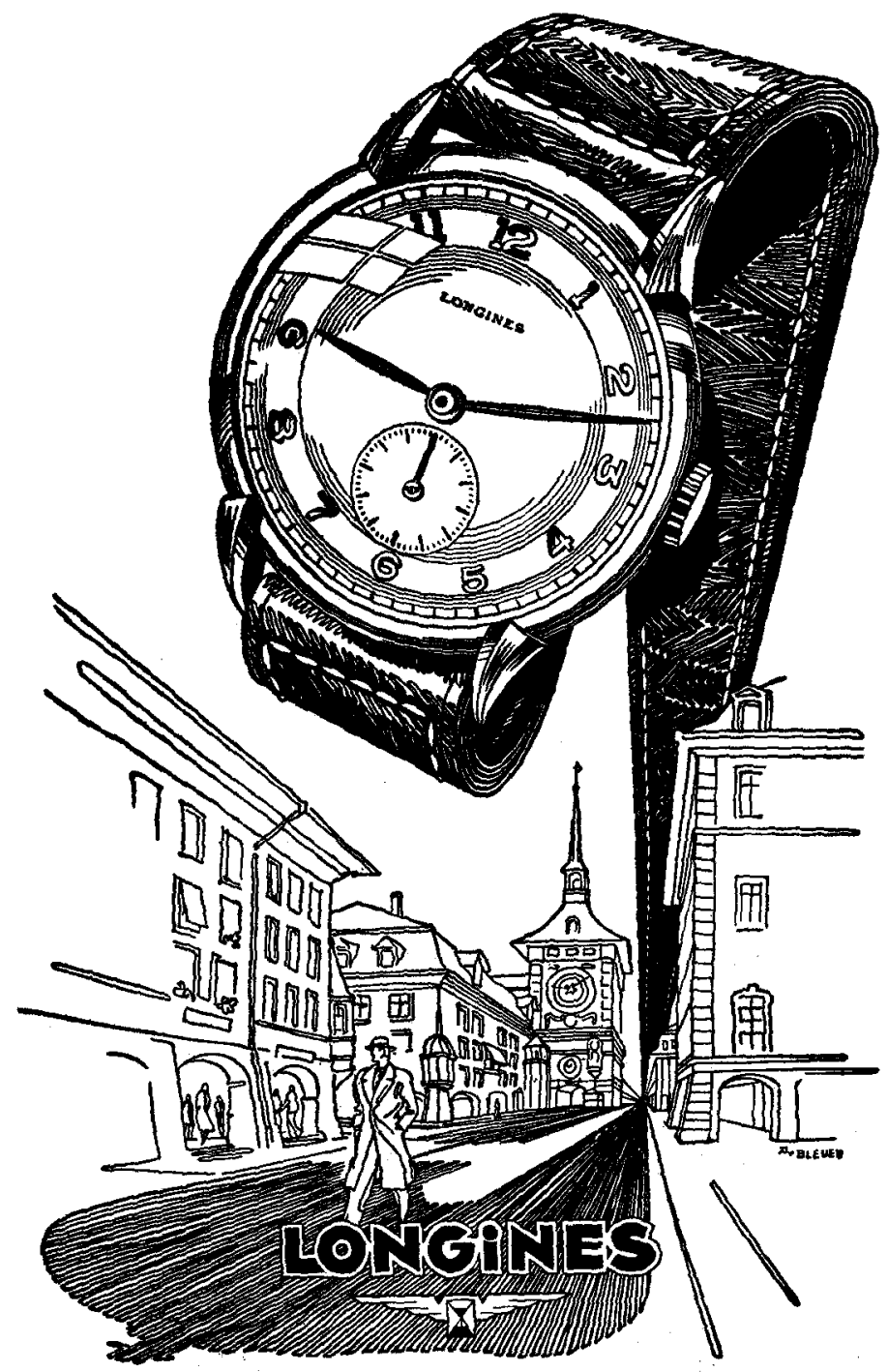




\section{IAIT SUISSE}

Marque

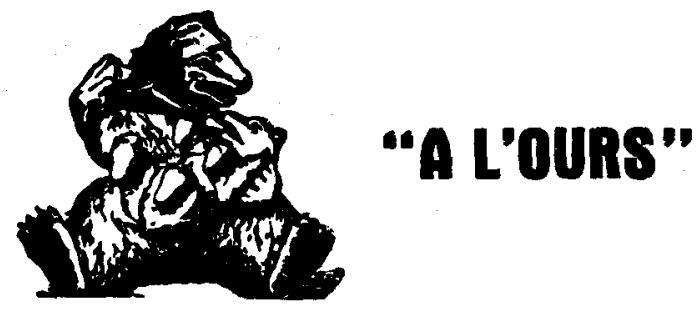

PREPARE PAR

SOCIÉTÉ LAITIĖRE DES ALPES BERNOISES

STALDEN, Emmenthal, SUISSE

Spécialités pour nourrissons, enfants et adultes :

LAITS CONCENTRÉS

"La Laitière " (sucré)

"Idéal " (non sucré)

\section{HAITS WN POUDRE}

"Pélargon", lait acidifié

“Nido», lait entier non sucré

\section{AMIDON, FARINES}

Soldor, amidon spécial

Farine Nestlé sans Lait

Farine Lactée Nestlé

\section{NESCAO}

Déjeuner fortifiant

chocolats, cacaOs

Nestlé, Peter, Cailler, Kohler

RXTRAITS DE CATE

Nescafé

Nescafé sans caféine

Nescoré

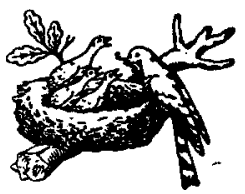

SOCIETÉ IES PRODUITS NESTLE S.A. VEVEY 


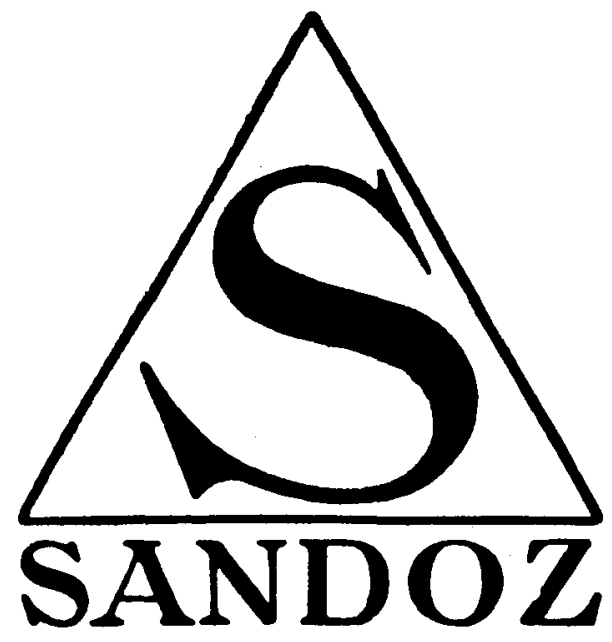

PRODUITS

\section{PHARMACEUTIQUES}

BALE (Suisse) 


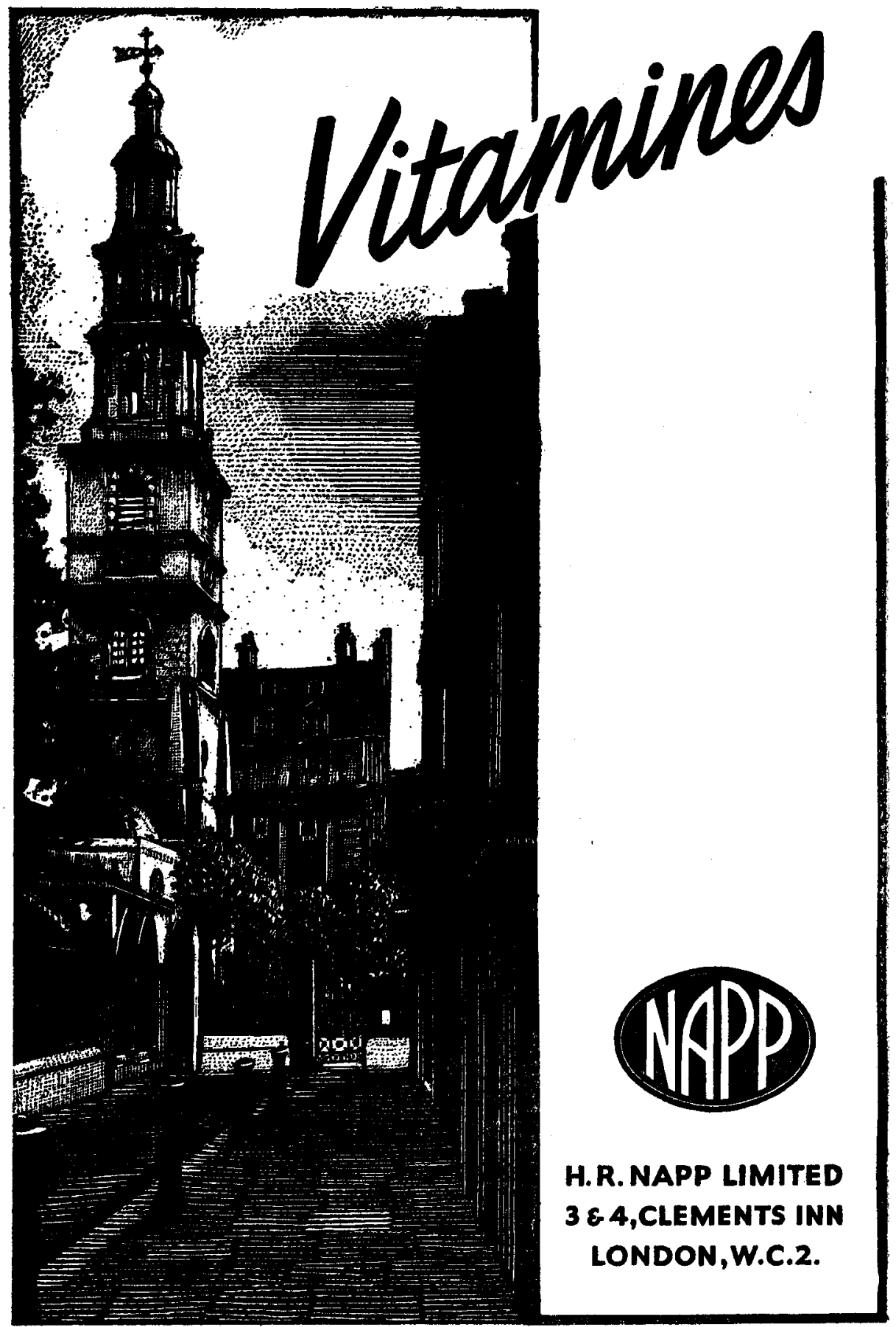

